\title{
Analysis and Comparison of Image Restoration Methods
}

\author{
Yubing Dong, Huaxun Zhang, and Mingjing Li \\ College of Electronics and Information Engineering, Changchun \\ University, Changchun, 130022, China
}

\begin{abstract}
The principles of inverse filtering, wiener filtering, and histogram equalization combined median filtering are introduced and studied. Three image restoration methods are compared in a variety of blur and noise conditions. Their own advantage and disadvantage are described. The simulation experiments of three methods for the motion blurred image with and without noise have been done by MATLAB. It is demonstrated that, in certain conditions, one restoration method is preferable to others.
\end{abstract}

Keywords:image degradation, image restoration, inverse filtering, wiener filtering, median filtering.

\section{Introduction}

The image is the human visual basic, gives specific and visual effects. Image in the acquisition, transmission and storage process will be subject to such as blurring, distortion, noise and other reasons, these reasons will make the image quality degradation. The purpose of image restoration is to rebuild original image from observation of its degraded image. It is studied widely as is the basis of image processing, model identification, machine vision, and so on. It has been applied on such fields as astronomical, remote sensing and medical image. The restoration of images is a hot research topic in the field of digital image processing, and the recovery of motion blurred or noise image is one of the important subjects of image restoration. As an important aspect of image processing, image restoration has got more and more attentions in recent years.

Many factors can cause the degradation, such as noise of sensor, not focus of camera, object movement, object illumination, light scatter. In cases like motion blur, it is possible to come up with a very good estimate of the actual blurring function and undo the blur to restore the original image. In cases where the image is corrupted by noise, the best we may hope to do is to compensate for the degradation it caused. Image restoration task is to find out the noise property and come up with a method to remove them and to find out the degradation function and perform the inverse process. The principal method of image restoration is firstly to construct the model of image degradation, then implement 
the image approximation according to the preceding model. According to unconstrained restoration, inverse filtering is used; according to constrained restoration, wiener filtering is used. Several of the methods used in the image processing world to restore images will be introduced and implemented in the paper.

The paper is organized as follows. In the next section, we propose the degradation function and model that we research in this paper, and some definitions and assumptions are given. In Section 3, two common restoration methods are introduced. Combining histogram equalization and median filtering, a new image restoration method is presented. Section 4 presents experimental results. Finally, we conclude our paper in section 5.

\section{Estimating the degradation function}

The degradation process is modeled as a degradation function. $H$ is the degradation function with some knowledge, $\eta(x, y)$ is the additive noise term with some knowledge, the object is to obtain an estimate $f(x, y)$ of the original image. The objective of restoration is to obtain an estimate $\hat{f}(x, y)$ of the original image. The estimate image $\hat{f}(x, y)$ is as close as possible to the original input image. The more $\mathrm{H}$ and $\eta$ are known, the closer $\hat{f}(x, y)$ will be to $f(x, y)$. If $\mathrm{H}$ is a linear, position-invariant process, then the degraded image model is given in the spatial domain by Eq.1 or in frequency domain by Eq.2 or in vector form by Eq.3. Where $\eta(x, y)$ is the additive noise term, $h(x, y)$ is called as Point Spread Function (PSF). A true image $f(x, y)$ is estimated from a degraded image $g(x, y)$ based on prior knowledge of $\operatorname{PSF} h(x, y)$ and the statistical properties of noise $\eta(x, y)$.

$$
\begin{gathered}
g(x, y)=h(x, y) * f(x, y)+\eta(x, y) \\
G(u, v)=H(u, v) F(u, v)+\eta(u, v) \\
g=H f+\eta
\end{gathered}
$$

A mathematical model of motion blur will be derived. If $\mathrm{T}$ is the duration of the exposure, the blurred image is obtained by Eq.4. Where $x_{o}(t)$ and $y_{o}(t)$ are the time varying components of motion in the $x$ - direction and $y$-direction.

$$
g(x, y)=\int_{0}^{T} f\left[x-x_{0}(t), y-y_{0}(t)\right] d t
$$

The spatial noise may be considered random variables characterized by a probability density function. For example, Gaussian noise' mathematical tractability in both spatial and frequency domains, this model is used frequently. 
The noise image is obtained by Eq.5. Where $z$ is gray level (Gaussian random variable), $u$ is the mean of average value of $\mathrm{z}$, and $\sigma$ is standard deviation.

$$
p(z)=\frac{1}{\sqrt{2 \pi \sigma}} e^{-(z-u)^{2} / 2 \sigma^{2}}
$$

\section{Image restoration methods}

\subsection{Inverse filtering}

If a good model of the blurring function is created, the quickest and easiest way to restore that is by inverse filtering. If $G(u, v)$ is divided by $H(u, v)$ to get an estimate of $F(u, v)$, then equation 6 is get. This is called direct inverse filtering.

$$
\hat{F}(u, v)=\frac{G(u, v)}{H(u, v)}=F(u, v)+\frac{N(u, v)}{H(u, v)}
$$

If $H(u, v)$ has zero or very small value, the $N(u, v) / H(u, v)$ can easily dominate the estimate. Through this method, an image assuming a known blurring function is looked. Restoration is good when noise is not present and not so good when it is. The inverse filtering is a restoration technique for deconvolution, i.e., when the image is blurred by a known low-pass filter, it is possible to recover the image by inverse filtering or generalized inverse filtering. However, inverse filtering is very sensitive to additive noise. The approach of reducing degradation at a time allows us to develop a restoration algorithm for each type of degradation and simply combine them.

\subsection{Wiener filtering.}

The Wiener filtering executes an optimal trade off between inverse filtering and noise smoothing. It removes the additive noise and inverts the blurring simultaneously. The Wiener filtering is optimal in terms of the mean square error. In other words, it minimizes the overall mean square error in the process of inverse filtering and noise smoothing. The Wiener filtering is a linear estimation of the original image. The approach is based on a stochastic framework. The orthogonal principle implies that the Wiener filter in Fourier domain can be expressed as follows Eq.7. Where $S_{f}(u, v)=|F(u, v)|^{2}, S_{n}(u, v)=|N(u, v)|^{2}$ are respectively power spectra of the original image and the additive noise, and $H(u, v)$ is the blurring filter.

$$
H_{W}(u, v)=\frac{H^{*}(u, v)}{|H(u, v)|^{2}+S_{n}(u, v) / S_{f}(u, v)}
$$


It is easy to see that the Wiener filter has two separate parts, an inverse filtering part and a noise smoothing part. It not only performs the de-convolution by inverse filtering (high-pass filtering) but also removes the noise with a compression operation (low-pass filtering). Image restoration using wiener filtering is implemented, which provides us with the optimal trade-off between de-noising and inverse filtering. The result is in general better than with straight inverse filtering.

\subsection{A new image restoration method.}

Combining histogram equalization and median filtering, a new image restoration method is proposed. The median filter is a nonlinear digital filtering technique, often used to remove noise. For an even number of entries, there is more than one possible median, see median for more details. Where $W=\left(W_{m n}\right)\left(W_{m n}=1\right.$ or 0$)$ is expressed as a filter window, the center value of the filter window $(\mathrm{m}, \mathrm{n})$ is $(0,0)$, and $\left\{f(x, y)(x, y) \in I^{2}\right\}$ is expressed as the image gray value of each point. The restoration image is obtained by Eq.8. $\hat{f}=\operatorname{Med}\{f(x, y)\}=\operatorname{Med}\left\{f(x+m, y+n) \mid W_{m n}=1, \quad(x, y) \in I^{2}\right\}(8)$

Histogram equalization is a method in image processing of contrast adjustment using the image's histogram. This method usually increases the global contrast of many images, especially when the usable data of the image is represented by close contrast values. Through this adjustment, the intensities can be better distributed on the histogram. The method is useful in images with backgrounds and foregrounds that are both bright or both dark. A key advantage of the method is that it is a fairly straightforward technique and an invertible operator. The transformation function is Eq.9. Where $f(x, y)$ is the input image, $\hat{f}(x, y)$ is the processed image, and $\mathrm{T}$ is an operator on $\mathrm{f}$ defined over some neighborhood of $(x, y)$.

$$
\hat{f}(x, y)=T[f(x, y)]
$$

\section{Experimental results}

In this paper, a car image is the research object. Through MATLAB simulation soft, the experiment using three image restoration methods are done in noise conditions. The simulation experiment results are shown in Fig. 1. The effects of images restoration are the best using median filtering combined histogram equalization. 


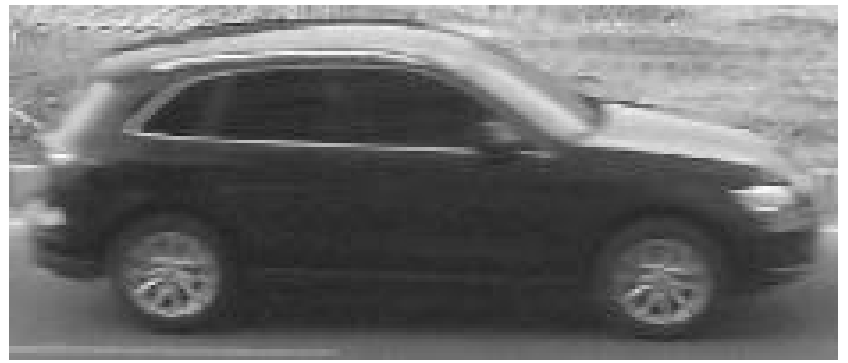

(a)Original image

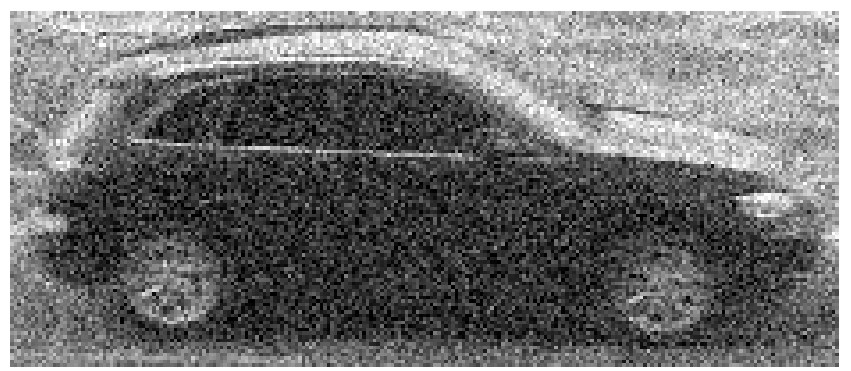

(b) Degradation image caused by noise

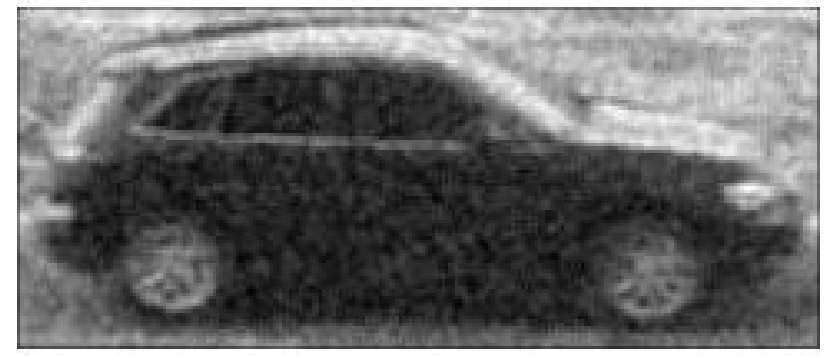

(c) Inverse filtering

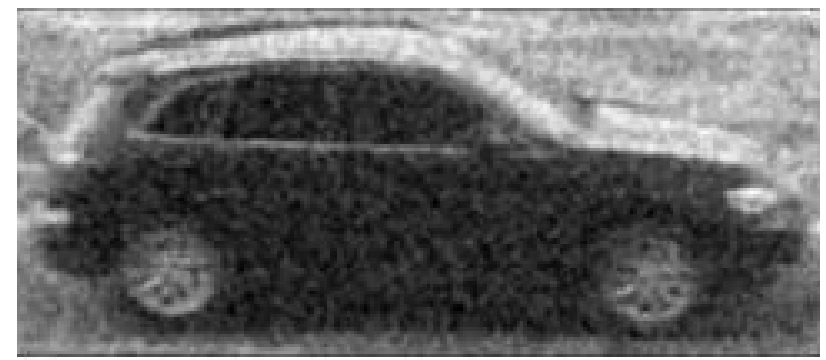

(d) Wiener filtering 


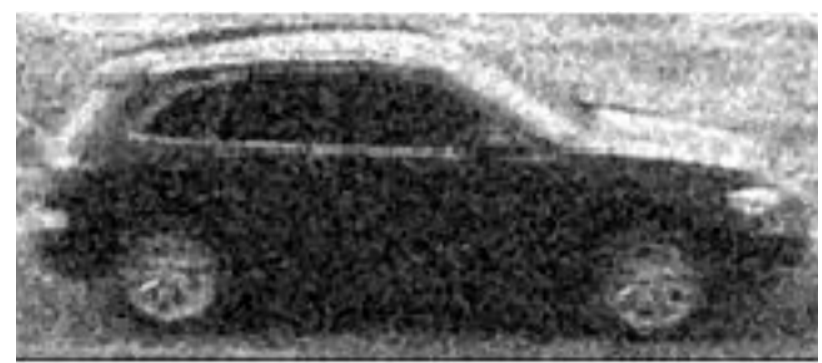

(e) New method

In motion blurred conditions, the simulation experiments are carried on, which the effects are shown in Fig. 2. Using inverse filtering, the effect of image restoration is the best.

Fig.1: Restoration image in noise condition

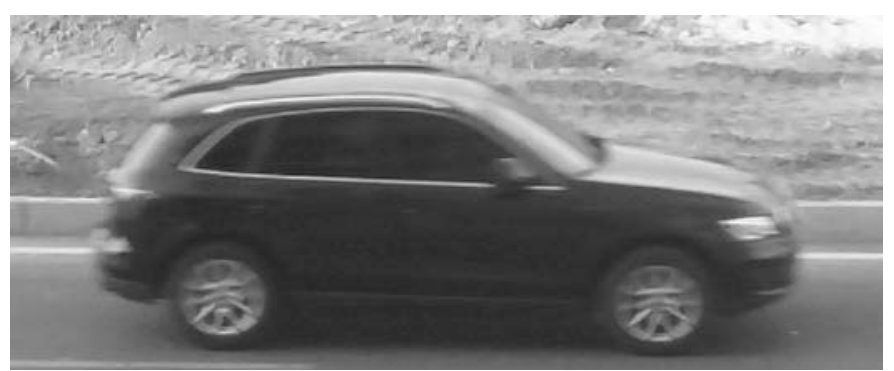

(a)Original image

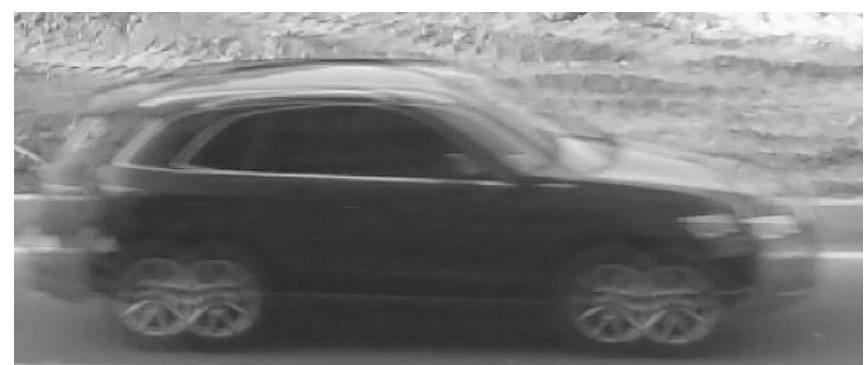

(b) Degradation image caused by motion blurred 


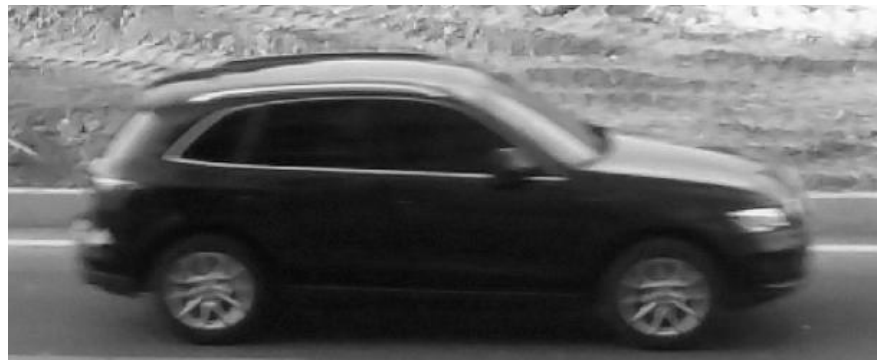

(c) Inverse filtering

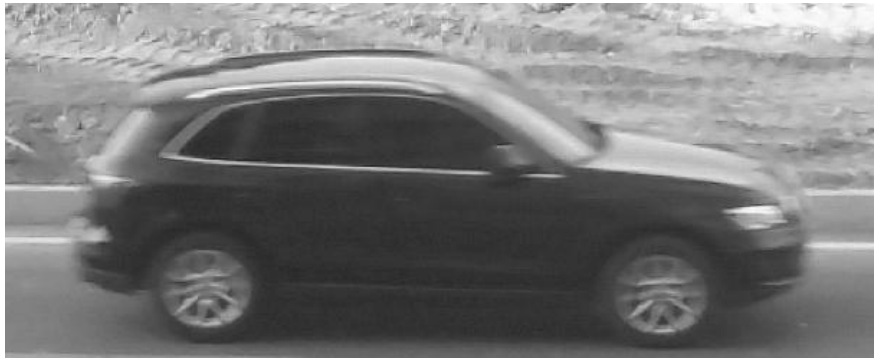

(d) Wiener filtering

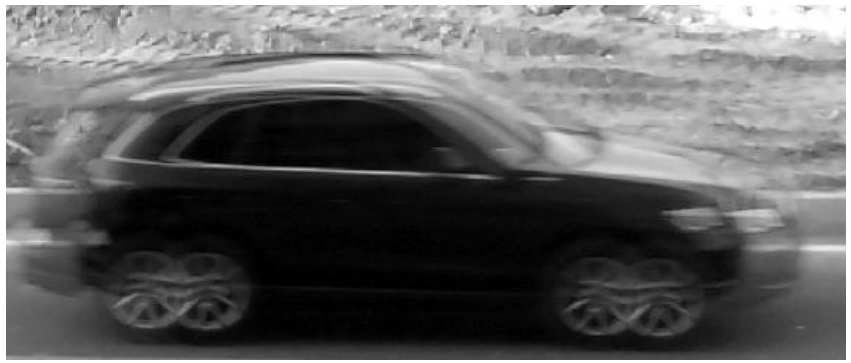

(e) New method

Fig.2: Restoration image in motion blurred condition

\section{Conclusions}

The present paper is mainly concerned with the restoration problem. The degradation functions of motion blurred and noise are introduced. Three image restoration methods are introduced, compared and simulated by MATLAB. The result is demonstrated that, in certain conditions, one restoration method is preferable to others. 


\section{Acknowledgements}

This work was financially supported by scientific research fund of Jilin provincial education department (2014, the number: 302), Project name is "Research on the key technology of portable target recognition system based on multi-sensors fusion”.

\section{References}

[1] Wu X J, Wang R S, Wang C. Regularized image restoration based on adaptively selecting parameter and operator. In: The 17th IEEE International Conference on Pattern Recognition, Cambridge, United Kingdom, Vol. 3 (6), pp. 662-665, 2004.

[2] Zhang Jianjun and Wang Qin. A new regularization method for bi-level image restoration[C]. International Conference on Audio, Language and Image Processing, pp.955-958, 2008.

[3] Yonina C. Eldar. Nonideal sampling and interpolation from noisy observation in shift-invariant spaces[C]. IEEE Trans. Signal processing. Vol.54, 2006.

[4] Lam E Y. Blind bi-level image restoration with iterated quadratic programming [J].IEEE Trans Circuits and Systems-II: Express Briefs, Vol. 54(36), pp. 52-55,2007.

[5] Wenjing Jia, Huaifeng Zhang, and Xiangjian He, Region-based license plate detection, Journal of Network and Computer Applications, Vol. 30(8), pp. 1324-1328, 2007.

[6] Tao Xun. A motion-blurred image restoration algorithm based on block registration, Computer Applications and Software. Vol. 28(2), pp. 246-249, 2011.

[7] Huang T, Yang G, and Tang G, A fast two-dimensional median filtering algorithm, IEEE Transactions on Acoustics, Speech and Signal Processing, Vol. 27, pp. 13-16, 2003.

[8] Bapeswara Rao V.V and Rao K.S, A new algorithm for real-time median filtering, IEEE Transactions on Acoustics, Speech and Signal Processing, Vol. 34, pp. 1674-,2003. 